\title{
ALP bouncing and LDH normalization in bone metastatic castration-resistant prostate cancer patients under therapy with Enzalutamide: an exploratory analysis
}

\author{
Katrin Schlack ${ }^{1}$, Laura-Maria Krabbe ${ }^{1}$, Kambiz Rahbar ${ }^{2}$, Karoline Isenberg ${ }^{1}$, Axel Semjonow ${ }^{1}$, \\ Andres Jan Schrader ${ }^{1}$, Martin Boegemann ${ }^{1}$ \\ ${ }^{1}$ Department of Urology, Prostate Center, University of Muenster Medical Center, Muenster, Germany; ${ }^{2}$ Department of Nuclear Medicine, \\ University of Muenster Medical Center, Muenster, Germany \\ Contributions: (I) Conception and design: K Schlack, M Boegemann; (II) Administrative support: M Boegemann, K Rahbar; (III) Provision of \\ study materials or patients: K Schlack, M Boegemann, A Semjonow, AJ Schrader, K Isenberg; (IV) Collection and assembly of data: K Schlack, M \\ Boegemann, K Isenberg; (V) Data analysis and interpretation: K Schlack, M Boegemann, AJ Schrader, LM Krabbe; (VI) Manuscript writing: All \\ authors; (VII) Final approval of manuscript: All authors. \\ Correspondence to: Katrin Schlack. Department of Urology, Prostate Center, University of Muenster Medical Center, Albert-Schweitzer-Campus 1, \\ GB A1, D-48149 Muenster, Germany. Email: katrin.schlack@ukmuenster.de.
}

Background: In bone metastatic castration-resistant prostate cancer (bmCRPC) treated with Enzalutamide commonly used prostate-specific antigen (PSA) can be misleading since initial PSA-flares may occur. In other therapies, bouncing of alkaline phosphatase (ALP-bouncing) was shown to be a promising surrogate for survival outcome. Low lactate dehydrogenase $(\mathrm{LDH})$ is usually associated with better outcome. We evaluated the prognostic ability of ALP-bouncing, LDH, PSA, and the combination of these markers after initiation of Enzalutamide.

Methods: Eighty-nine patients with bmCRPC and dynamic changes of PSA, LDH and ALP were analyzed. ALP-bouncing, an increase after therapy start followed by a decline below baseline during the first 8 weeks, LDH-normalization and PSA-decline were analyzed regarding their association with survival using KaplanMeier analyses and uni- and multivariate (UV and MV) Cox-regression models.

Results: In Kaplan-Meier analysis a PSA-decline >50\%, LDH-normalization and ALP-bouncing were associated with longer median progression-free survival (PFS) with 7 [95\% confidence interval (CI): 4.2-9.8] vs. 3 (2.3-3.7) months for PSA-decline (log-rank $\mathrm{P}<0.01), 6$ (4.1-8) vs. 2 (1.2-2.8) for LDH-normalization $(\mathrm{P}<0.01)$ and $8(0-16.3)$ vs. $3(1.9-4.1)$ for ALP-bouncing $(\mathrm{P}=0.01)$. Analysis of overall survival (OS) showed similar, not for all parameters significant, results with 17 (11.7-22.3) vs. 12 (7.0-17.1) months for PSA $(\mathrm{P}=0.35), 17$ (13.2-20.8) vs. 7 (5.8-8.2) for LDH-normalization $(\mathrm{P}<0.01)$ and 19 (7.9-30.1) vs. 12 (7.7-16.3) for ALP-bouncing ( $\mathrm{P}=0.32)$. In UV analysis, ALP-bouncing [hazard ratio (HR): 0.5 (0.3-1.0); $\mathrm{P}=0.02$ ], PSA-decline $>50 \%$ [HR: $0.5(0.3-0.7)$; $\mathrm{P}<0.01$ ] and LDH-normalization [HR: $0.4(0.2-0.6) ; \mathrm{P}<0.01]$ were significantly associated with longer PFS. For OS, LDH-normalization significantly prognosticated longer survival [HR: $0.4(0.2-0.6) ; \mathrm{P}<0.01$ ]. In $\mathrm{MV}$ analysis, LDH-normalization was associated with a trend towards better OS [HR: 0.5 (0.2-1.1); P=0.09]. Comparing ALP-bouncing, LDH-normalization and PSAdecline with a PSA-decline alone, Kaplan-Meier analysis showed significantly longer PFS [11 (0.2-21.8) vs. 4 (0-8.6); $\mathrm{P}=0.01]$ and $\mathrm{OS}[20$ (17.7-22.3) vs. 8 (0.3-15.7); $\mathrm{P}=0.02]$ in favor of the group presenting with the beneficial dynamics of all three markers. In UV analysis, the presence of favorable changes in the three markers was significantly associated with longer PFS [HR: $0.2(0.1-0.7)$; $\mathrm{P}<0.01]$ and OS [HR: $0.3(0.1-0.8)$; $\mathrm{P}=0.02]$.

Conclusions: ALP-bouncing and LDH-normalization may add to identification of bmCRPC-patients with favorable prognosis under Enzalutamide. 
Keywords: Alkaline phosphatase (ALP); bone metastatic castration-resistant prostate cancer (bmCRPC); Enzalutamide; lactate dehydrogenase (LDH); prostate-specific antigen (PSA)

Submitted Jul 25, 2020. Accepted for publication Nov 30, 2020.

doi: $10.21037 /$ tau-20-1117

View this article at: http://dx.doi.org/10.21037/tau-20-1117

\section{Introduction}

In recent years significant progress in therapy strategies for metastatic castration-resistant prostate cancer (mCRPC) has been made. When resistance to androgen-deprivation therapy (ADT) occurs, prostate cancer can be treated with chemotherapy (Docetaxel or Cabazitaxel), Sipuleucel-T, Radium-223 or next generation ADT drugs (Abiraterone acetate or Enzalutamide) (1-8). Amongst these, Enzalutamide, a targeted $2^{\text {nd }}$ generation androgen-receptor (AR) inhibitor, is widely used as standard of care due to its increase of overall survival (OS) and low toxicity $(1,2)$. A triple mechanism of action including inhibition of binding to the $\mathrm{AR}$ and consequently inhibition of the AR entering the nucleus as well as inhibition of the AR binding to the DNA results in potent AR-pathway blockage (9).

In metastatic disease the most common site of metastases, next to loco-regional lymph nodes, are the bones (10). A spread to the skeleton increases the risk of complications including fractures of the bones and subsequently death $(11,12)$. Hence, early measurement of treatment success, particularly the identification of response or failure in asymptomatic patients, can be clinically difficult but, nevertheless, is important.

Currently, treatment success can be assessed with the help of several laboratory biomarkers, clinical factors [e.g., eastern co-operative oncology group (ECOG) performance status] and imaging [computed tomography (CT), magnetic resonance imaging (MRI), and bone scintigraphy]. The most readily available measurements of treatment success presumably are blood-based biomarkers. Several biomarkers have previously been described including prostate-specific antigen (PSA), lactate dehydrogenase (LDH), circulating tumor cells (CTCs) and, particularly in bone metastatic disease, alkaline phosphatase (ALP) (3,13-21). Amongst these, PSA is most widely used and, under therapy with Enzalutamide, a decline was shown to be associated with improved survival outcomes (22). However, there are several limitations since dynamic changes of PSA-values are not always straightforward. During the first 3-6 months a PSAflare can occur before a potential delayed decline may be registered (18,23-26). CTC counts at baseline and changes under therapy were shown to be prognostic for survival outcomes (27). However, CTC enumeration is limited in patients with low tumor burden and can significantly deviate depending on the site of metastasis (28). Additionally, CTCassays are costly and not broadly available.

$\mathrm{LDH}$ is a prognostic biomarker in most malignancies and associated with better survival outcomes when in the normal range or normalizing under therapy (29-31). Despite having been shown to be a surrogate for favourable prostate cancer outcome when baseline levels are low, it remains to be an unspecific biomarker $(17,21,32)$. In contrast, ALP, as a more bone-related parameter, seems to have prognostic potential in bone metastatic CRPC (bmCRPC) patients under therapy with Docetaxel, Radium-223 or Abiraterone acetate (16,17,33-35). An interesting phenomenon under therapy of bmCRPC called ALP-bouncing seems to occur under very early treatment of effective antihormonal therapy and is followed by a significant decline below baseline level after 8 weeks of therapy (17). It was first described in a patient undergoing bilateral orchiectomy for treatment of bmCRPC (36). Recently, Mikah et al. reported that ALP-bouncing may be an early promising surrogate for favourable survival in bmCRPC patients under Abiraterone-therapy (17).

In summary, several biomarkers seem to have potential to prognosticate outcome under therapy in bmCRPC-patients but the information on their prognostic value is still limited. In this study, we aimed to investigate the possible surrogate potential of LDH and ALP-bouncing along with PSA and the potential benefit of the combination of all three parameters under therapy with Enzalutamide. We present the following article in accordance with the REMARK reporting checklist (available at http://dx.doi.org/10.21037/tau-20-1117).

\section{Methods}

\section{Patients}

For this study, we retrospectively reviewed 99 patients receiving Enzalutamide who presented at the Department of 


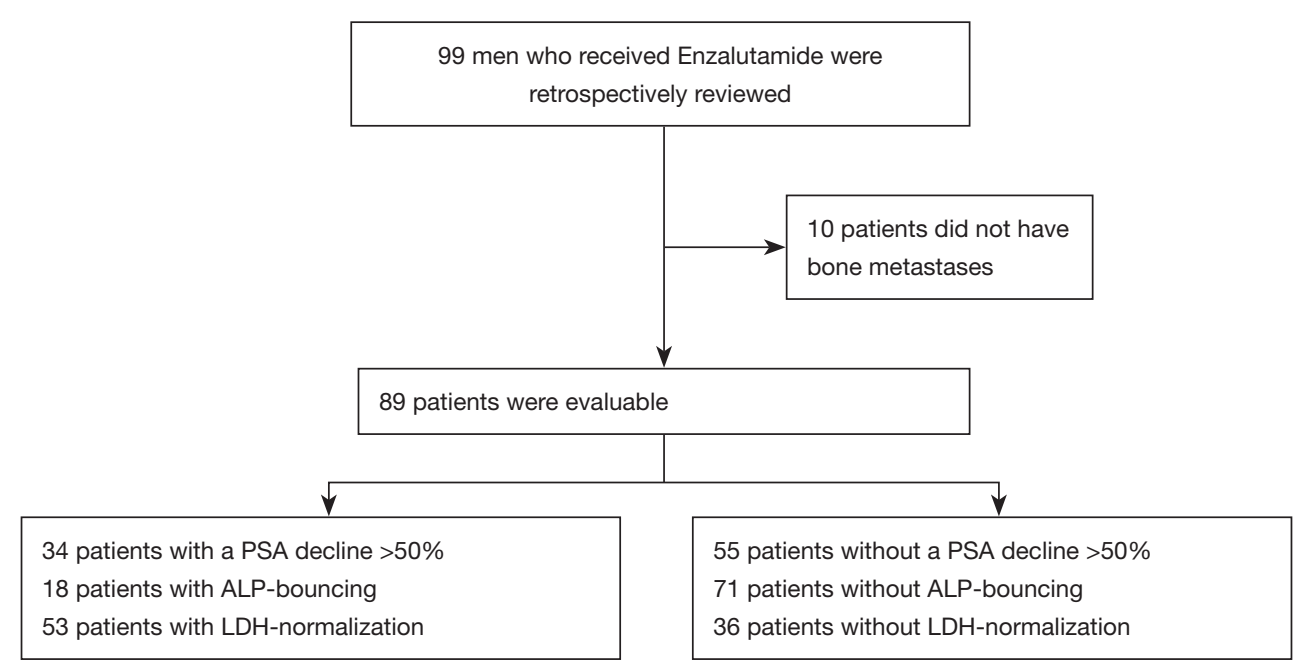

Figure 1 A CONSORT flow diagram of the process through the phases of patient selection and the results regarding the respective number of patients with changes of PSA, ALP and LDH. PSA, prostate-specific antigen; ALP, alkaline phosphatase; LDH, lactate dehydrogenase.

Urology of the University Hospital in Muenster, Germany between $07 / 2010$ and 11/2016. The process of patient selection is displayed in Figure 1. Before participating ethics committee-approval was granted (Ethikkomission der Aerztekammer Westfalen-Lippe und der Medizinischen Fakultaet der Westfaelischen Wilhelms-Universitaet Muenster: 2007-467-f-S) and all patients had given written informed consent. The study was conducted in accordance with the Declaration of Helsinki (as revised in 2013).

Since ALP as a biomarker in mCRPC is mainly related to bone metastases, patients with non-bone-metastatic disease were excluded from the final analysis, resulting in 89 evaluable subjects. At the last follow-up 21 patients were alive. All patients had confirmed bmCRPC, defined by prostate cancer working group 3 (PCWG 3 criteria) (37) and received Enzalutamide in an either pre- or post-chemotherapy (Docetaxel) setting and partly after treatment with Abiraterone. Pre-treated patients after Abiraterone or Docetaxel had prior documented progressive disease (PD). There were no patients receiving concomitant Radium-223 treatment.

The 61 patients receiving bone antiresorptive therapy started at least 3 months prior to initiation of Enzalutamide and stayed on a stable dose for the treatment phase. The other patients did not receive antiresorptive agents at all.

All patients treated with Enzalutamide prior to Docetaxel chemotherapy were asymptomatic or mildly symptomatic, not requiring opiates and presenting with a baseline pain level of no more than 3 out of 10 on the numeric-rating-scale.
All patients presented the day before the start of Enzalutamide therapy to have blood drawn for baseline analysis. After treatment initiation, follow-up examinations (evaluation of pain level and ECOG) took place after 2 and 4 weeks and four-weekly thereafter. Following blood draw PSA-, LDH-, and ALP-levels were measured in serum samples on the same day. In human plasma ALP and LDH were measured with ADVIA 1800 Clinical Chemistry System and PSA with ADVIA Centaur XP Immunoassay System (Siemens Healthcare, Erlangen, Germany). Total values and dynamic changes of the three markers were documented. Normalized LDH was defined as either value in the normal range throughout the whole study period or normalization under therapy after elevation above the upper limit of normal at therapy start. Rising ALP during the first 2-8 weeks after initiation of therapy followed by a decline below pre-treatment levels was defined as ALP-bouncing. Minimal changes were not taken into consideration due to biological reasons or measurement variance.

Progression-free survival (PFS) was defined as either PSA progression or radiographic progression. PSA progression was defined as confirmed increase of $25 \%$ or greater from baseline in case of no prior PSA decline and an absolute increase of $2 \mathrm{ng} / \mathrm{mL}$ or more from nadir (PCWG 3 criteria) (37).

When no PD was suspected imaging, consisting of CTor MRI-scans of thorax, abdomen and pelvis as well as bone scans, was not routinely performed. When biochemical progression occurred, or progression was presumed by 
clinical factors, soft tissue metastases were evaluated by CTand MRI-scans. Bone metastases were assessed by additional bone scans. A total of 13 patients in our cohort were treated within the Enzalutamide registration trials (AFFIRM and PREVAIL) (1,2). Even without evidence for PD, these patients were systematically followed up with 12 -weekly imaging according to the study protocol. For all patients PD was defined according to RECIST 1.1 criteria for crosssectional imaging (38) and by PCWG 3 criteria for bone scans (37). The assessment of current response status i.e., complete remission (CR), partial remission (PR), stable disease (SD) or PD took place at each routinely planned visit and was independently performed by experienced physicians and experts in the field of mCRPC. For the classification of response status, general condition (ECOG), pain or laboratory constellations (PSA) as well as imaging were taken into account.

\section{Statistical methods}

The statistical assessment was performed with SPSSStatistics V.24 (IBM Inc., Armonk, NY, USA).

The descriptive statistics are reported as medians with interquartile ranges (IQRs) or 95\% confidence intervals (CIs) for continuous variables and as frequencies and populations for categorical variables.

Regarding the differences between ALP-bouncing $v s$. no bouncing and LDH-normalization $v s$. no normalization, as well as PSA increase $v s$. decline, survival analysis was performed using Kaplan-Meier-Analyses (KMA). Uniand multivariate (UV and MV) analyses of changes of the different biomarkers were done using Cox-regressionmodels. Hazard ratios (HRs) are given with 95\% CI. All reported $\mathrm{P}$ values are two-sided and statistical significance was assumed with a $\mathrm{P} \leq 0.05$.

\section{Results}

\section{Characteristics of the study group}

The whole cohort consisted of 89 men of whom 36 (40.4\%) received Enzalutamide in a pre-chemotherapy and 53 $(59.6 \%)$ in a post-chemotherapy setting. Demographics of the cohort are presented in Table 1. Abiraterone had previously been administered in 66 (74.2\%) of the patients. Sixty-one of our patients (68.5\%) received antiresorptive therapy [i.e., Zoledronic acid $(31.5 \%)$ or Denusomab (37.1\%)]. ECOG performance status was $0-3$ in our cohort,
Table 1 Characteristics of patients with bmCRPC on Enzalutamide

\begin{tabular}{|c|c|}
\hline Variables & All \\
\hline Patients, n (\%) & $89(100.0)$ \\
\hline Age (years), median (IQR) & $70(62.5-76)$ \\
\hline Lymph node metastases, n (\%) & $55(61.8)$ \\
\hline Visceral metastases, n (\%) & $14(15.7)$ \\
\hline Pre-chemotherapy, n (\%) & $36(40.4)$ \\
\hline Post-chemotherapy, n (\%) & $53(59.6)$ \\
\hline Enzalutamide post Abiraterone, $\mathrm{n}(\%)$ & $66(74.2)$ \\
\hline Patients died, n (\%) & $68(76.4)$ \\
\hline Antiresorptive therapy, $\mathrm{n}(\%)$ & $61(68.5)$ \\
\hline Zoledronic acid & $28(31.5)$ \\
\hline Denosumab & $33(37.1)$ \\
\hline \multicolumn{2}{|l|}{ Best clinical outcome, $\mathrm{n}(\%)$} \\
\hline CR & $1(1.1)$ \\
\hline PR & $32(36.0)$ \\
\hline SD & $32(36.0)$ \\
\hline PD & $24(27.0)$ \\
\hline \multicolumn{2}{|l|}{ ECOG (all), n (\%) } \\
\hline 0 & $40(44.9)$ \\
\hline 1 & 35 (39.3) \\
\hline 2 & $13(14.6)$ \\
\hline 3 & $1(1.1)$ \\
\hline Gleason-Score $\geq 8, \mathrm{n}(\%)$ & $50(56.2)$ \\
\hline Median ALP at baseline (U/L), median (IQR) & $155(104.5-318.5)$ \\
\hline Median LDH at baseline (U/L), median (IQR) & $274(219-348.5)$ \\
\hline Median PSA at baseline ( $\mathrm{ng} / \mathrm{mL})$, median (IQR) & $176(45.4-506)$ \\
\hline PSA-decline $\geq 50 \%$, n (\%) & $34(38.2)$ \\
\hline PSA-decline $\geq 90 \%, \mathrm{n}(\%)$ & $12(13.5)$ \\
\hline LDH at baseline > UNL, n (\%) & $60(67.4)$ \\
\hline LDH-normalization, n (\%) & $53(59.6)$ \\
\hline ALP rising at 12 weeks Enzalutamide, n (\%) & $19(21.3)$ \\
\hline ALP-bouncing, $n(\%)$ & $18(19.8)$ \\
\hline $\begin{array}{l}\text { PSA decline } \geq 50 \% \text { alone, no ALP-bouncing, no } \\
\text { LDH-normalization, } n(\%)\end{array}$ & $10(11.2)$ \\
\hline $\begin{array}{l}\text { PSA decline } \geq 50 \% \text { AND LDH-normalization } \\
\text { AND ALP-bouncing, } n(\%)\end{array}$ & $10(11.2)$ \\
\hline
\end{tabular}

bmCRPC, bone metastatic castration-resistant prostate cancer; $I Q R$, interquartile range; $C R$, complete remission; $P R$, partial remission; SD, stable disease; PD, progressive disease; ALP, alkaline phosphatase; LDH, lactate dehydrogenase; PSA, prostate-specific antigen; UNL, upper normal limit; ECOG, eastern co-operative oncology group. 


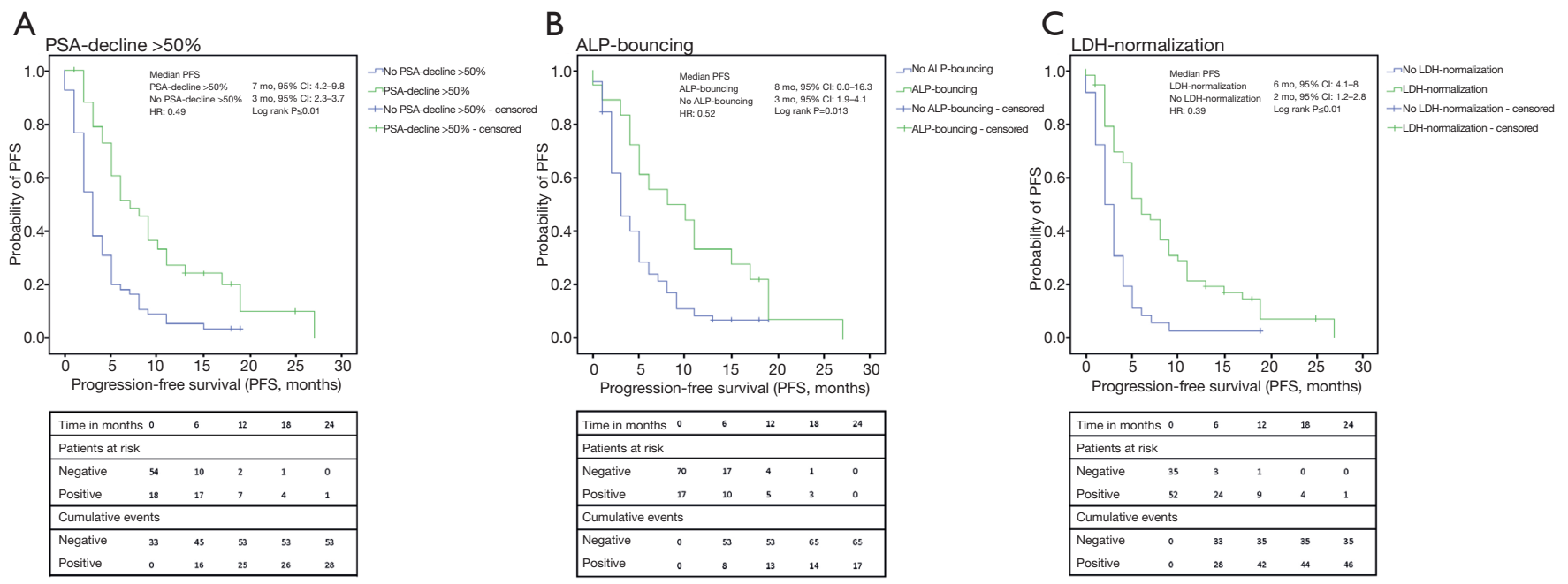

Figure 2 Kaplan-Meier analyses of PFS. (A) The analysis of PFS resulted in a median PFS of 7 months for patients with a PSA-decline of $50 \%$ or more compared to 3 months when patients did not show that decline ( $\mathrm{P} \leq 0.01)$; (B) considering ALP, median PFS was 8 vs. 3 months in favor of the presence of ALP-bouncing $(\mathrm{P}=0.013)$; $(\mathrm{C})$ patients with LDH-normalization showed a median PFS of 6 months while the opposite led to a PFS of 2 months $(\mathrm{P} \leq 0.01)$. PFS, progression-free survival; PSA, prostate-specific antigen; ALP, alkaline phosphatase; LDH, lactate dehydrogenase; HR, hazard ratio; CI, confidence interval.

most of our patients showed an ECOG of 0 (44.9\%) or 1 (39.3\%). An ECOG level of 2 was present in $14.6 \%$ and 3 in $1.1 \%$ of the patients.

The median follow-up was 13 (IQR: 6.5-19.0) months. The median time to first imaging was 3 (IQR: $3-6)$ months for the 67 (75.3\%) patients who underwent CT- or MRIand bone-scans. The median time on Enzalutamidetherapy was 6 (IQR: 3-12.5) months with 7 (7.9\%) patients with ongoing therapy at the time of analysis. No dose modifications were necessary for any patient. The median age of the patients was 70 (IQR: 62.5-76.0) years. At the beginning of Enzalutamide therapy, lymphonodal and visceral metastases were present in $61.8 \%$ and $15.7 \%$ of patients, respectively. An unfavorable Gleason-Score of $\geq 8$ at initial diagnosis was present in $56.2 \%$. Median baseline levels were 176 (IQR: 45.4-506.0) $\mathrm{ng} / \mathrm{mL}$ for PSA, 274 (IQR: 219.0-348.5) U/L for LDH, and 155 (IQR: 104.5318.5) U/L for ALP (Table 1).

\section{Change of PSA, LDH and ALP as prognostic markers}

Results of the Kaplan-Meier analyses are given in Figures 2-4. Parameters with favorable change of values were associated with improved survival outcomes.

PSA-decline $>50 \%$, ALP-bouncing and LDHnormalization were associated with a PFS of 7 (95\%
CI: 4.2-9.8) vs. 3 (2.3-3.7) months (log-rank $\mathrm{P}<0.01), 8$ (0-16.3) vs. 3 (1.9-4.1) months $(\mathrm{P}=0.01)$ and $6(4.1-8)$ vs. 2 $(1.2-2.8)$ months $(\mathrm{P}<0.01)$, respectively. Despite statistical relevance was only reached for LDH-normalization, analysis of OS showed similar results with $17(11.7-22.3)$ vs. 12 (7.0-17.1) months for PSA-decline $>50 \%(\mathrm{P}=0.35)$, 19 (7.9-30.1) vs. 12 (7.7-16.3) months for ALP-bouncing $(\mathrm{P}=0.32)$ and $17(13.2-20.8)$ vs. 7 (5.8-8.2) months for LDH-normalization $(\mathrm{P}<0.01)$.

We divided the patients into a group with PSA-decline $>50 \%$, LDH-normalization, and ALP-bouncing $(\mathrm{n}=10) v s$. a group with PSA-decline $>50 \%$ only $(\mathrm{n}=10)$. By combining the information of these markers, the Kaplan-Meier analysis showed significantly longer PFS [11 (0.2-21.8) vs. 4 (0-8.6) months; $\mathrm{P}=0.01]$ and $\mathrm{OS}[20(17.7-22.3)$ vs. 8 $(0.3-15.7)$ months; $\mathrm{P}=0.02]$ in favor of the group with three beneficial markers.

The relevant biomarkers that showed statistically significant improved PFS in Kaplan-Meier analyses were also identified as prognosticators for better survival outcomes in UV. Results are displayed in Table 2. Considering PFS, ALP-bouncing, PSA-decline $>50 \%$, LDH-normalization, no elevated LDH at baseline, no rising ALP after 12 weeks, Enzalutamide before Abiraterone or chemotherapy as well as ECOG $>1$ were statistically significant [HR: 0.5 (95\% CI: 0.3-1.0); 
A

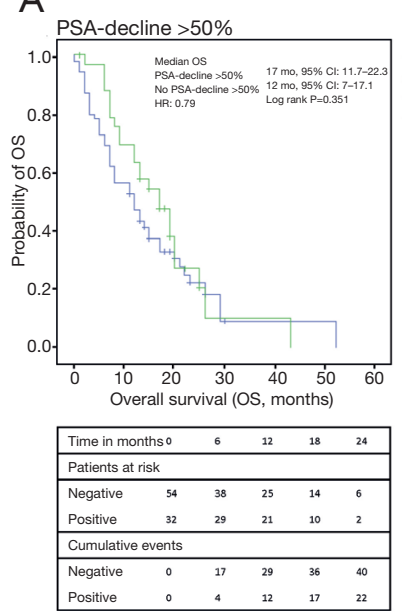

B

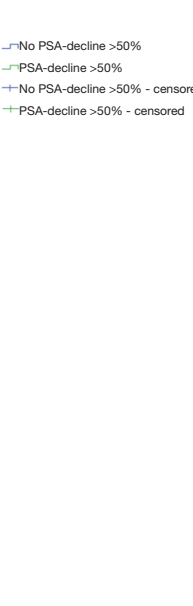

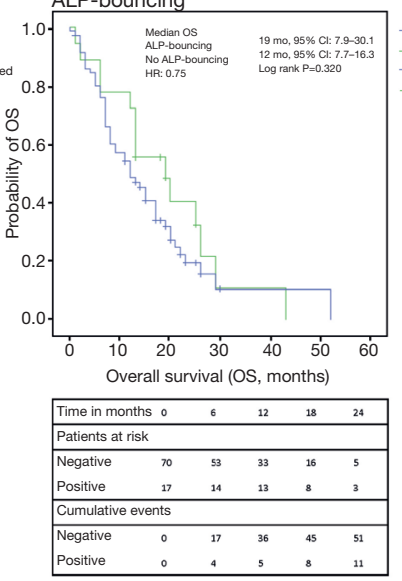

C

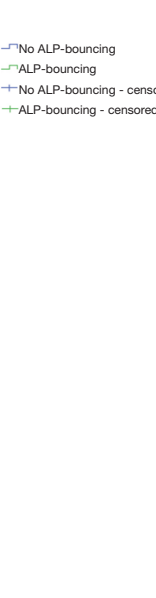

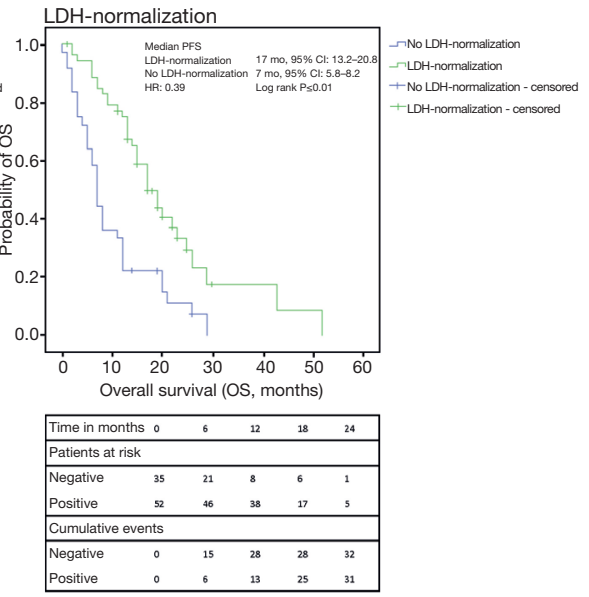

Figure 3 Kaplan-Meier analyses of OS. (A) The analysis of OS resulted in a median OS of 17 months for patients with a PSA-decline of $50 \%$ or more compared to 12 months when patients did not show that decline ( $\mathrm{P} \leq 0.01)$; (B) patients with ALP-bouncing had a median OS of 19 compared to 12 months for patients who did not present with ALP-bouncing $(\mathrm{P}=0.32)$; (C) the presence of LDH-normalization was associated with a better OS of 17 months whereas no LDH-normalization resulted in an OS of 7 months $(\mathrm{P} \leq 0.01)$. OS, overall survival; PSA, prostate-specific antigen; ALP, alkaline phosphatase; LDH, lactate dehydrogenase; HR, hazard ratio; CI, confidence interval.
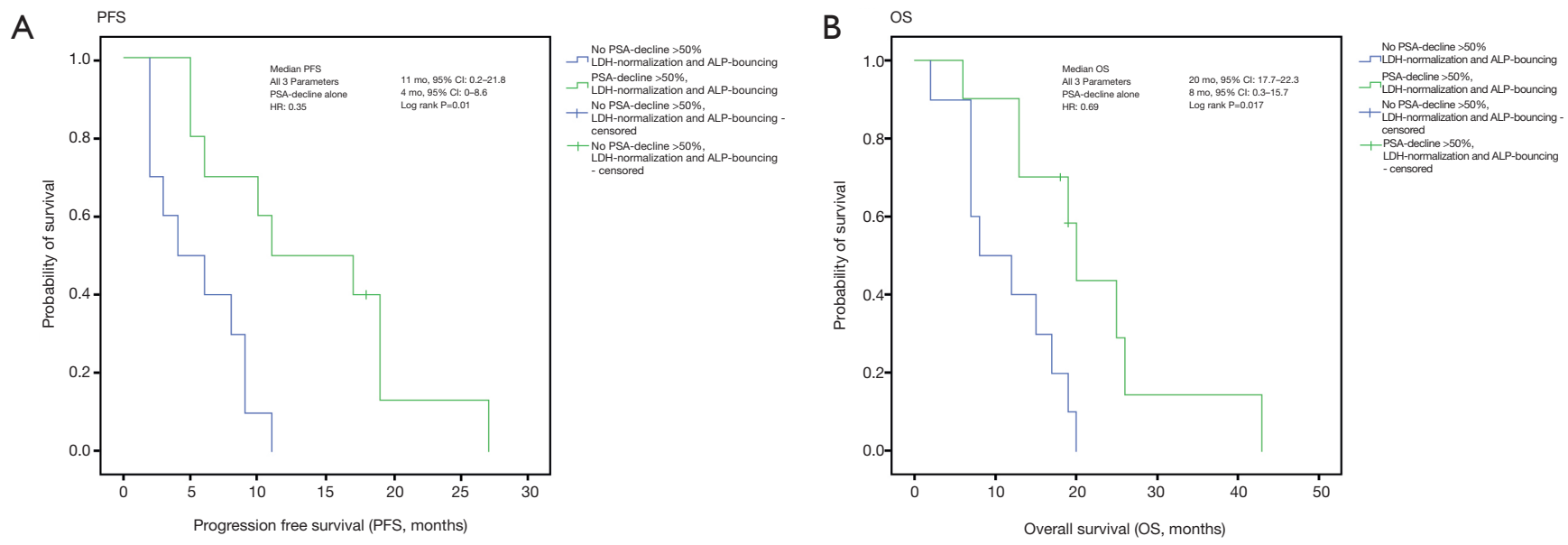

\begin{tabular}{|lccccc|}
\hline Time in months & 0 & 6 & 12 & 18 & 24 \\
\hline Patients at risk & & & & & \\
\hline Negative & 10 & 4 & 0 & 0 & 0 \\
Positive & 10 & 7 & 5 & 3 & 1 \\
\hline Cumulative events & & & & & \\
\hline Negative & 0 & 6 & 10 & 10 & 10 \\
Positive & 0 & 3 & 5 & 6 & 8 \\
\hline
\end{tabular}

\begin{tabular}{|lccccc|}
\hline Time in months & 0 & 6 & 12 & 18 & 24 \\
\hline Patients at risk & & & & & \\
\hline Negative & 10 & 6 & 4 & 1 & 0 \\
Positive & 10 & 9 & 7 & 6 & 2 \\
\hline Cumulative events & & & & & \\
\hline Negative & 0 & 4 & 6 & 9 & 10 \\
Positive & 0 & 1 & 3 & 3 & 6 \\
\hline
\end{tabular}

Figure 4 Kaplan-Meier analyses for the changes of the biomarkers collectively. (A) For patients with favorable changes in all of the three markers (PSA-decline $>50 \%$, ALP-bouncing and LDH-normalization) $\mathrm{PFS}$ was 11 vs. 4 months ( $\mathrm{P}=0.01)$ compared to the group of patients with a PSA-decline $>50 \%$ alone; (B) for patients with favorable changes in all of the three markers (PSA-decline $>50 \%$, ALP-bouncing and LDH-normalization) OS was 20 vs. 8 months $(\mathrm{P}=0.017)$ compared to the group of patients with a PSA-decline $>50 \%$ alone. PSA, prostatespecific antigen; ALP, alkaline phosphatase; LDH, lactate dehydrogenase; PFS, progression-free survival; OS, overall survival; HR, hazard ratio; CI, confidence interval. 
Table 2 UV analyses of significant biomarkers for OS in 89 bmCRPC-patients on Enzalutamide therapy

\begin{tabular}{|c|c|c|c|c|}
\hline Variables & \multicolumn{2}{|c|}{ PFS } & \multicolumn{2}{|c|}{ OS } \\
\hline ALP-bouncing & & 0.02 & & 0.33 \\
\hline Yes & 1 (reference) & & 1 (reference) & \\
\hline No & $0.5(0.3-1.0)$ & & $0.8(0.4-1.4)$ & \\
\hline Yes & 1 (reference) & & 1 (reference) & \\
\hline No & $0.5(0.3-0.9)$ & & $0.6(0.3-1.0)$ & \\
\hline Enzalutamide before chemotherapy & & 0.02 & & $<0.01$ \\
\hline Yes & 1 (reference) & & 1 (reference) & \\
\hline Yes & 1 (reference) & & 1 (reference) & \\
\hline No & $0.5(0.3-0.7)$ & & $0.8(0.5-1.3)$ & \\
\hline LDH normalized anytime & & $<0.01$ & & $<0.01$ \\
\hline Yes & 1 (reference) & & 1 (reference) & \\
\hline No & $0.4(0.2-0.6)$ & & $0.4(0.2-0.6)$ & \\
\hline ALP rising after 12 weeks & & 0.01 & & 0.31 \\
\hline No & 1 (reference) & & 1 (reference) & \\
\hline Yes & $2.1(1.2-3.7)$ & & $1.4(0.7-2.5)$ & \\
\hline Yes & $0.7(0.4-1.1)$ & & $0.5(0.3-0.8)$ & \\
\hline ALP decline after 12 weeks & & 0.07 & & 0.62 \\
\hline No & 1 (reference) & & 1 (reference) & \\
\hline Yes & $1.6(0.9-2.6)$ & & $1.2(0.7-2.0)$ & \\
\hline Visceral metastases & & 0.96 & & 0.66 \\
\hline No & 1 (reference) & & 1 (reference) & \\
\hline Yes & $1.0(0.6-1.8)$ & & $1.2(0.6-2.4)$ & \\
\hline $\mathrm{ECOG}>1$ & & $<0.01$ & & $<0.01$ \\
\hline Yes & 1 (reference) & & 1 (reference) & \\
\hline No & $0.3(0.2-0.5)$ & & $0.2(0.1-0.5)$ & \\
\hline
\end{tabular}

UV, univariate; OS, overall survival; bmCRPC, bone metastatic castration-resistant prostate cancer; PFS, progression-free survival; HR, hazard ratio; Cl, confidence interval; ALP, alkaline phosphatase; AA, abiraterone acetate; PSA, prostate-specific antigen; LDH, lactate dehydrogenase; ECOG, eastern co-operative oncology group. 
Table 3 MV analyses of biomarkers for OS in 89 bmCRPC-patients on Enzalutamide-therapy

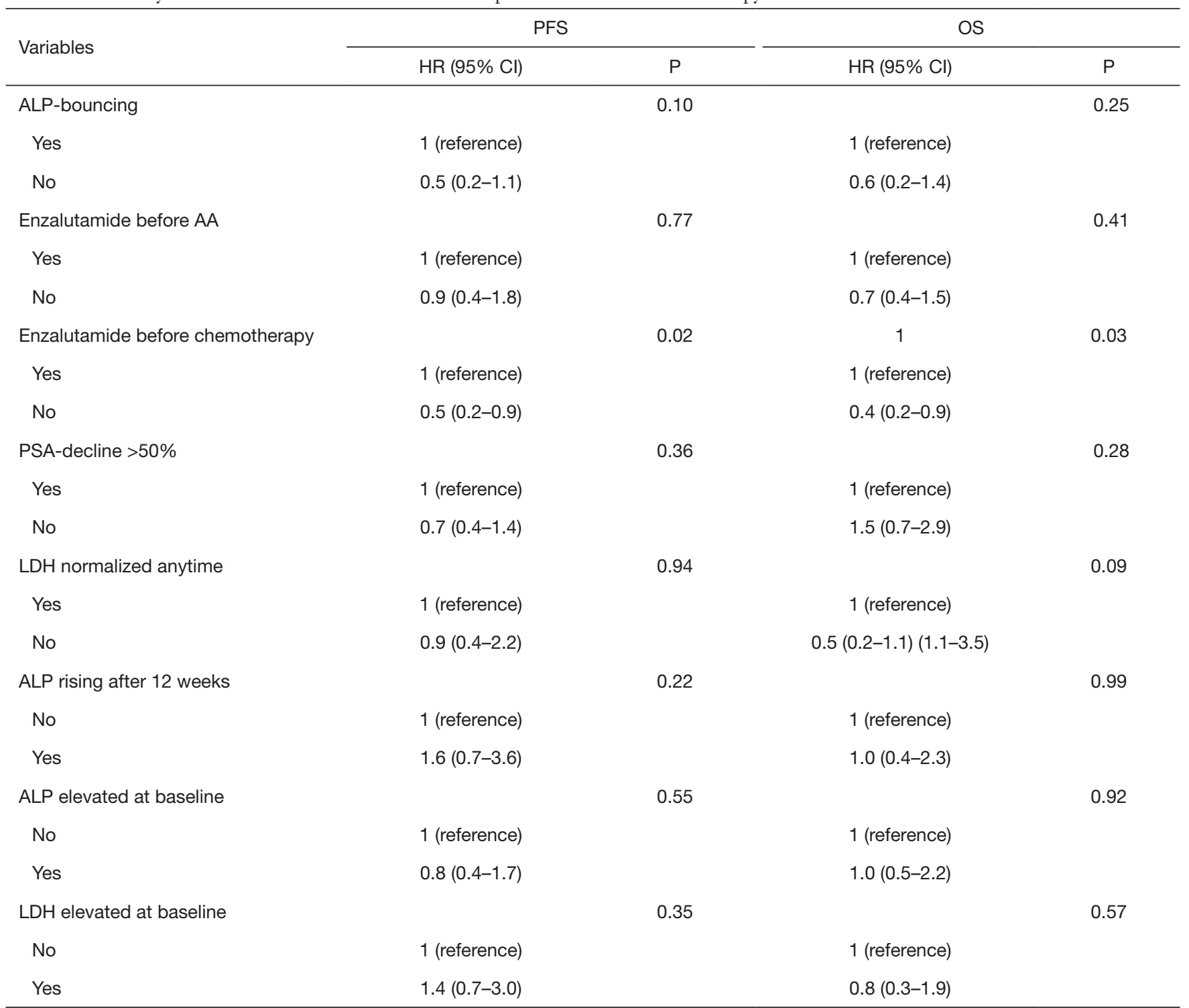

MV, multivariate; OS, overall survival; bmCRPC, bone metastatic castration-resistant prostate cancer; PFS, progression-free survival; HR, hazard ratio; $\mathrm{Cl}$, confidence interval; ALP, alkaline phosphatase; AA, abiraterone acetate; PSA, prostate-specific antigen; LDH, lactate dehydrogenase.

$\mathrm{P}=0.02 ; 0.5$ (95\% CI: 0.3-0.7); log-rank $\mathrm{P}<0.01 ; 0.4$ (95\% CI: $0.2-0.6) ; \mathrm{P}<0.01 ; 0.5,0.3-0.8, \mathrm{P}<0.01 ; 2.1$ (95\% CI: $1.2-3.7) ; \mathrm{P}=0.01 ; 0.5$ (95\% CI: $0.3-0.9) ; \mathrm{P}<0.01 ; 0.6$ (95\% CI: 0.4-0.9); $\mathrm{P}=0.02,0.3$ (95\% CI: $0.2-0.5$ ); $\mathrm{P} \leq 0.01$, respectively]. In the analysis of OS, normal ALP at baseline, LDH-normalization during treatment, the application of Enzalutamide before chemotherapy, and ECOG $>1$ showed the highest level of significance towards prolonged survival [HR: 0.4 (95\% CI: 0.2-0.6); P<0.01; 0.5 (95\% CI: 0.3-0.8);
$\mathrm{P}<0.01 ; 0.5$ (95\% CI: 0.3-0.8); $\mathrm{P}<0.01,0.2$ (95\% CI: 0.1$0.5) ; \mathrm{P} \leq 0.01]$.

In $M V$ only the intake of Enzalutamide before chemotherapy remained a significant prognosticator for both PFS [HR: 0.5 (95\% CI: 0.2-0.9); $\mathrm{P}=0.02$ ] and OS [HR: 0.4 (95\% CI: 0.2-0.9); $\mathrm{P}=0.03)$ ]. The results are given in Table 3. A trend towards prolonged survival was shown for ALP-bouncing [HR: 0.5 (95\% CI: 0.2-1.1); $\mathrm{P}=0.10$ ] for PFS and for LDH-normalization [HR: 0.5 (95\% CI: 
Table 4 UV analysis of the combined biomarkers for PFS and OS in 20 bmCRPC-patients on Enzalutamide-therapy

\begin{tabular}{|c|c|c|c|c|}
\hline Variables & \multicolumn{2}{|l|}{ PFS } & \multicolumn{2}{|l|}{ OS } \\
\hline ALP-bouncing AND LDH-normalization AND PSA-decline & $0.23(0.1-0.7)$ & 0.01 & $0.26(0.1-0.8)$ & 0.02 \\
\hline PSA-decline alone & $4.4(1.4-13.4)$ & 0.01 & $3.8(1.3-11.5)$ & 0.02 \\
\hline
\end{tabular}

UV, univariate; PFS, progression-free survival; OS, overall survival; bmCRPC, bone metastatic castration-resistant prostate cancer; HR, hazard ratio; $\mathrm{Cl}$, confidence interval; ALP, alkaline phosphatase; $\mathrm{LDH}$, lactate dehydrogenase; PSA, prostate-specific antigen.
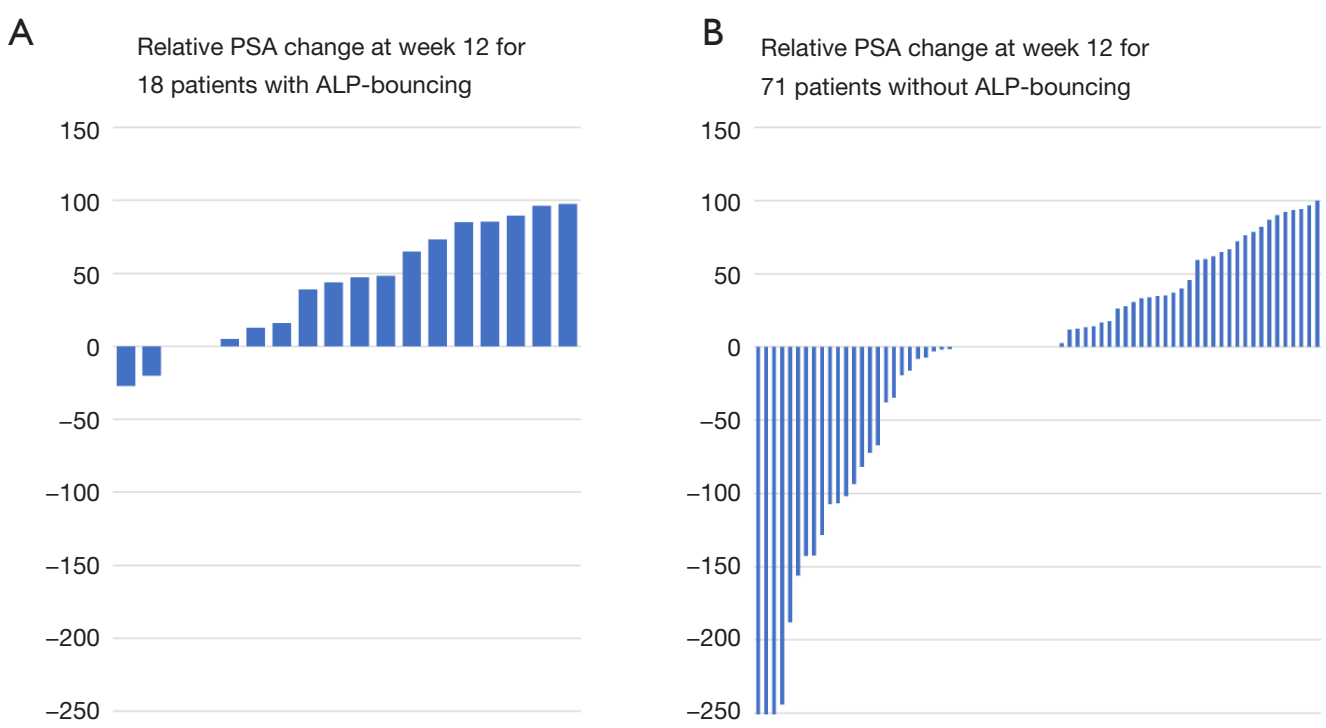

Figure 5 Relative PSA change at week 12. (A) Patients with ALP-bouncing showed less frequently a PSA-decline and a lower PSA response compared to the subgroup of patients without ALP-bouncing; (B) patients without ALP-bouncing showed more often a PSA-decline and a better PSA response. PSA, prostate-specific antigen; ALP, alkaline phosphatase.

\section{2-1.1); $\mathrm{P}=0.09$ ] for OS.}

Due to the smaller sample sizes, after separating the patients into a group with ALP-bouncing, LDHnormalization and PSA-decline $>50 \%$ and a group with PSA-decline $>50 \%$ alone, only UV was performed. We saw that the combined markers and their favorable changes were associated with better survival outcomes (Table 4). Patients with a PSA-decline $>50 \%$ only had a significant worse PFS [HR: 4.40 (95\% CI: 1.4-13.4); $\mathrm{P}=0.01]$ and OS [HR: 3.8 (95\% CI: 1.3-11.5); $\mathrm{P}=0.02$ ] as compared to the combination of favorable markers [PFS HR: 0.2 ; (95\% CI: 0.1-0.7); $\mathrm{P}=0.01$, OS HR: 0.3 (95\% CI: $0.1-0.8$ ), $\mathrm{P}=0.02$, respectively].

The relative PSA change is displayed in Figure 5 showing that patients with ALP-bouncing had less frequently a PSA-decline and a lower PSA response compared to the subgroup of patients without ALP-bouncing.

\section{Discussion}

Currently, there are few biomarkers available that may help to prognosticate the outcome under a given therapy in mCRPC. The interpretation of a single marker in clinical routine is difficult and rarely done separately from other factors like ECOG or imaging.

Considering patients with $\mathrm{mCRPC}$, a large proportion (80-90\%) presents with bone metastases $(39,40)$. In these patients, especially under early treatment during the first three to 6 months, a "bone-flare" can occur and might lead to misinterpretation and incorrect classification as PD (41). This dilemma becomes particularly relevant in oligo- or asymptomatic patients where clinical factors like pain level or ECOG cannot be included as additional information (40).

A similar phenomenon can be observed for PSA. PSA is the most common biomarker used for therapy monitoring 
in mCRPC patients. According to the above mentioned "bone-flare", PSA may likewise rise under early therapy before declining. In our cohort 9 patients (10.1\%) showed this "PSA-flare phenomenon". In all of these cases PSA declined after 3 months and was followed by a PR or SD. The PSA-flare has been described under different therapies with a duration varying from 3 to 6 months $(18,23-26,42)$. Since the early rise of PSA might be a sign of tumor cell death with subsequent circulatory release of PSA and therefore does not necessarily represent PD, current guidelines do not recommend to discontinue treatment based on PSA-information alone (43). On the contrary, a PSA-decline usually goes along with treatment success. Therefore, guidelines recommend to determine the percentage of patients showing a PSA-decline $>50 \%$ after 12 weeks of therapy within clinical trials (44). In this context, another study even showed that a PSA response, defined as a decline $\geq 30 \%$, at a very early stage of treatment with either Abiraterone or Enzalutamide is strongly associated with clinical outcome (45). A large cohort of 1,133 patients was evaluated in which a PSA-decline $\geq 30 \%$ at week 4 was associated with longer OS. This underlines that declining PSA values usually indicate treatment success. Nevertheless, within this analysis, after adjustment for known prognostic parameters such as ALP, LDH and neutrophile-to-lymphocyte ratio, a PSA-decline $\geq 30 \%$, at week 4 reduced the incidence of mortality compared to patients without PSA change but no statistically significant difference was found for patients with a $\geq 25 \%$ increase compared to the group of patients that did not show PSA changes (45). Even though a PSA-flare was uncommon in this study, one fact is very noticable; unquestionably a PSA decline indicates better outcome, but an increase does not exclude the possibility of a response. For this reason, within the registration trials for Enzalutamide, PREVAIL and AFFIRM, treatment discontinuation was discouraged based on an increase of PSA-level alone $(1,2)$. In everyday practice with difficulties due to the above mentioned "bone-flare", PSA often remains the only information for physicians to decide whether to stop or to continue the treatment. Within this scenario our aim was to find possible markers or parameters that might help to guide treatment.

Alternative biomarkers have been investigated for a long time. In contrast to PSA, CTCs do not show changes comparable to a "flare-phenomenon". Therefore, these parameters could be ideal markers as surrogate for survival outcomes (46). CTC counts at baseline and changes under therapy were shown to be prognostic for survival outcomes (27).
Recently, a prospective multicentre study showed that rising CTCs under therapy with Enzalutamide or Abiraterone were significantly associated with worse PFS and OS (47). However, the translation of CTC enumeration into clinical routine currently remains hard to do due to high costs, limited sensitivity and absence of easily available assays and is therefore mainly limited to clinical trials $(13,46)$.

$\mathrm{LDH}$, on the contrary, is a broadly available prognostic biomarker reflecting higher tumor burden or proliferation activity and hence aggressiveness of cancer (48) and when elevated was shown to be associated with a higher risk of death in prostate cancer patients (49). However, LDH remains an unspecific biomarker since higher levels are associated with worse survival outcomes in most malignancies and for example occur in inflammatory diseases as well without being able to differentiate between inflammation or neoplasm (29-31,50). Therefore, taking into account $\mathrm{LDH}$-values as a single parameter is not leading straightforward.

ALP, as a more bone-related parameter, seems to have prognostic potential in bmCRPC patients under therapy with Docetaxel, Radium-223 or Abiraterone acetate (16,17,33-35). During the first weeks of therapy, ALPbouncing seems to be a promising surrogate for favourable survival in bmCRPC patients receiving Abiraterone (17). To our knowledge, the prognostic value of this phenomenon for patients receiving Enzalutamide has not been investigated so far. Compared to PSA, with a relevant amount of flares or insignificant decline, the authors of the previously mentioned analysis concluded that ALP provides earlier information on response to therapy than PSA. For both the PSA-decline $\geq 50 \%$ and ALP-bouncing the outcome was very compareable (17). This is in line with our results. Regarding the differences, other than the administered treatment, it becomes apparent that ALPbouncing occurred more often $(40.4 \%$ vs. $19.8 \%)$ in the study of Mikah et al. A possible reason for this may be the higher number of patients pretreated with Abiraterone in our Enzalutamide cohort (74.2\%) compared to only 3 patients (3.6\%) who received Enzalutamide before Abiraterone within the other analysis. Further, the group of patients with ALP-bouncing showed less frequent PSA-decline in our collective compared to Mikah et al. potentially due to the same reason.

As previously described, the most challenging aspect during therapy of bmCRPC is the measurement of treatment success. Enzalutamide is often administered in patients with a lack of symptoms. Since initial "bone-flare" occurs 
in a large proportion of the cases early imaging can be misleading and should be avoided during the first 3 months of therapy $(40,51)$. Especially during this time a "PSA-flare" can be observed in some patients which leads into difficult decision making harbouring the possibility that the beneficial drug will be discontinued prematurely $(18,23-26,42)$. To prevent this, current guidelines recommend avoiding decision-making based on PSA-rise alone with the risk of unwillingly missing true disease progression (43).

In our study, we investigated dynamic changes of all of the three previously described and easily available biomarkers ALP, LDH and PSA during early Enzalutamide therapy to bridge the gap of the first 3 months of therapy with Enzalutamide when PSA and imaging might be misleading. According to current data and guidelines, our results show that PSA-decline $>50 \%$ as a single marker is a surrogate for longer PFS (43) whereas it does not improve OS (52). The occurrence of ALP-bouncing 2-8 weeks after initiation of therapy giving the advantage of earlier information was a prognosticator for better PFS as well. Additionally, normal or normalized values of LDH were a strong predictor for improved PFS and OS. However, none of the three parameters was shown to be an independent predictor for survival outcomes in our cohort. Therefore, we decided to combine the information we had by forming a group of patients where all markers showed favourable dynamics and a group with PSA-decline $>50 \%$ alone. Despite these subgroups being relatively small, the combination of these biomarker changes relevantly improved the prognostication of outcomes. In $\mathrm{UV}$, compared to the combination of all three beneficial biomarkers (PSA-decline, LDH-normalization, and ALPbouncing) the sole PSA-decline was associated with worse PFS and OS. This, of course, is a result of the artificial formation of two groups within the cohort of patients with PSA-decline usually being a beneficial marker.

Translation into clinical routine would appear premature but, from our perspective, taking ALP and LDH into consideration before discontinuing therapy in asymptomatic patients that might show "flare-phenomena" would be reasonable. This becomes especially true for the subgroup of patients with a PSA decline only since clinicians would expect that they respond to Enzalutamide. However, our data show that patients with a PSA decline alone and no other beneficial changes of LDH or ALP may be at risk of worse outcome despite favourable PSA-dynamics. This can be underlined by our finding that a PSA decline is not associated with an improved OS.
Unfortunately, our sample size was too small to combine a group of patients with a PSA-flare and the beneficial changes of LDH and ALP. Therefore, we could only show that there is an even better group within a group of patients with improved survival outcomes. Hence, we can only assume that the worse survival outcomes for PSA decline alone underline the possible role of LDH and ALP changes and that the additional information might help in cases of a PSA-flare.

Our study is limited due to its deficiencies inherent to a retrospective review and its relatively small collective. Furthermore, irregular imaging intervals could be a potential bias in the recording of PFS. However, eightynine patients are a large amount taking in account that they were consecutively recruited in a single center. By separating the patients into subgroups, the group sizes became relatively small. Therefore, larger prospective trials are warranted to validate the significance of our findings.

\section{Conclusions}

ALP-bouncing and LDH-normalization and the combination of these markers with PSA may add to identify bmCRPC patients with favorable prognosis as compared to PSA-reduction alone during treatment with Enzalutamide. Favorable changes in each of the three markers and of the combined markers are associated with improved survival outcomes.

\section{Acknowledgments}

The authors would like to thank Manfred Fobker for his helpful advice on technical issues in laboratory measurements and Corinna Schlack for her help in setting up the graphics format.

Funding: None.

\section{Footnote}

Provenance and Peer Review: This article was commissioned by the Guest Editor (Tilman Todenhöfer) for the series "Management of Advanced Genitourinary Malignancies" published in Translational Andrology and Urology. The article has undergone external peer review.

Reporting Checklist: The authors have completed the REMARK reporting checklist. Available at http://dx.doi. org/10.21037/tau-20-1117 
Data Sharing Statement: Available at http://dx.doi. org/10.21037/tau-20-1117

Peer Review File: Available at http://dx.doi.org/10.21037/ tau-20-1117

Conflicts of Interest: All authors have completed the ICMJE uniform disclosure form (available at http://dx.doi. org/10.21037/tau-20-1117). The series "Management of Advanced Genitourinary Malignancies" was commissioned by the editorial office without any funding or sponsorship. Dr. KS reports other from Astellas, during the conduct of the study; personal fees from Janssen, non-financial support from Astellas, non-financial support from Bayer, personal fees from AstraZeneca, personal fees from Pfizer, personal fees from Novartis, personal fees from EUSApharm, personal fees from Amgen, personal fees from Ipsen, personal fees from Merck, personal fees from MSD, personal fees from BMS, personal fees from Eisai, outside the submitted work. Dr. KR reports personal fees from Bayer Healthcare, personal fees from AAA, personal fees from $A B X$, personal fees from Janssen Cielag, personal fees from AMGEN, outside the submitted work. Dr. AS reports grants from Astellas, during the conduct of the study; other from Myriad, other from German Cancer Aid, other from Philips Healthcare, other from Proteomedix, personal fees from Janssen, personal fees from Ipsen, outside the submitted work. In addition, Dr. AS has a patent characterization of primary tumors (039PCT0735) issued. Dr. MB reports personal fees from Astellas, during the conduct of the study; grants and personal fees from Janssen, personal fees from Astellas, personal fees from Bayer, personal fees from AstraZeneca, personal fees from Sanofi, personal fees from Pfizer, personal fees from Novartis, personal fees from EUSApharm, personal fees from Amgen, personal fees from Ipsen, personal fees from Merck, personal fees from MSD, personal fees from BMS, personal fees from Eisai, personal fees from ABX, outside the submitted work. The authors have no other conflicts of interest to declare.

Ethical Statement: The authors are accountable for all aspects of the work in ensuring that questions related to the accuracy or integrity of any part of the work are appropriately investigated and resolved. The study was conducted in accordance with the Declaration of Helsinki (as revised in 2013). Ethics committee approval was granted by the Ethikkomission der Aerztekammer Westfalen-
Lippe und der Medizinischen Fakultaet der Westfaelischen Wilhelms-Universitaet Muenster (registration number: 2007-467-f-S) and all patients had given written informed consent.

Open Access Statement: This is an Open Access article distributed in accordance with the Creative Commons Attribution-NonCommercial-NoDerivs 4.0 International License (CC BY-NC-ND 4.0), which permits the noncommercial replication and distribution of the article with the strict proviso that no changes or edits are made and the original work is properly cited (including links to both the formal publication through the relevant DOI and the license). See: https://creativecommons.org/licenses/by-nc-nd/4.0/.

\section{References}

1. Beer TM, Armstrong AJ, Rathkopf DE, et al. Enzalutamide in metastatic prostate cancer before chemotherapy. N Engl J Med 2014;371:424-33.

2. Scher HI, Fizazi K, Saad F, et al. Increased survival with enzalutamide in prostate cancer after chemotherapy. $\mathrm{N}$ Engl J Med 2012;367:1187-97.

3. Berthold DR, Pond GR, Roessner M, et al. Treatment of hormone-refractory prostate cancer with docetaxel or mitoxantrone: relationships between prostate-specific antigen, pain, and quality of life response and survival in the TAX-327 study. Clin Cancer Res 2008;14:2763-7.

4. Ryan CJ, Smith MR, de Bono JS, et al. Abiraterone in metastatic prostate cancer without previous chemotherapy. N Engl J Med 2013;368:138-48.

5. Fizazi K, Scher HI, Molina A, et al. Abiraterone acetate for treatment of metastatic castration-resistant prostate cancer: final overall survival analysis of the COU-AA-301 randomised, double-blind, placebo-controlled phase 3 study. Lancet Oncol 2012;13:983-92.

6. Parker C, Nilsson S, Heinrich D, et al. Alpha emitter radium-223 and survival in metastatic prostate cancer. $\mathrm{N}$ Engl J Med 2013;369:213-23.

7. Kantoff PW, Higano CS, Shore ND, et al. Sipuleucel-T immunotherapy for castration-resistant prostate cancer. N Engl J Med 2010;363:411-22.

8. de Bono JS, Oudard S, Ozguroglu M, et al. Prednisone plus cabazitaxel or mitoxantrone for metastatic castrationresistant prostate cancer progressing after docetaxel treatment: a randomised open-label trial. Lancet 2010;376:1147-54.

9. Scher HI, Beer TM, Higano CS, et al. Antitumour activity 
of MDV3100 in castration-resistant prostate cancer: a phase 1-2 study. Lancet 2010;375:1437-46.

10. Hernandez RK, Wade SW, Reich A, et al. Incidence of bone metastases in patients with solid tumors: analysis of oncology electronic medical records in the United States. BMC Cancer 2018;18:44.

11. Smith MR, Saad F, Oudard S, et al. Denosumab and bone metastasis-free survival in men with nonmetastatic castration-resistant prostate cancer: exploratory analyses by baseline prostate-specific antigen doubling time. J Clin Oncol 2013;31:3800-6.

12. Fizazi K, Carducci M, Smith M, et al. Denosumab versus zoledronic acid for treatment of bone metastases in men with castration-resistant prostate cancer: a randomised, double-blind study. Lancet 2011;377:813-22.

13. de Bono JS, Scher HI, Montgomery RB, et al. Circulating tumor cells predict survival benefit from treatment in metastatic castration-resistant prostate cancer. Clin Cancer Res 2008;14:6302-9.

14. Scher HI, Heller G, Molina A, et al. Circulating tumor cell biomarker panel as an individual-level surrogate for survival in metastatic castration-resistant prostate cancer. J Clin Oncol 2015;33:1348-55.

15. Scher HI, Morris MJ, Basch E, et al. End points and outcomes in castration-resistant prostate cancer: from clinical trials to clinical practice. J Clin Oncol 2011;29:3695-704.

16. Sonpavde G, Pond GR, Berry WR, et al. Serum alkaline phosphatase changes predict survival independent of PSA changes in men with castration-resistant prostate cancer and bone metastasis receiving chemotherapy. Urol Oncol 2012;30:607-13.

17. Mikah P, Krabbe LM, Eminaga O, et al. Dynamic changes of alkaline phosphatase are strongly associated with PSAdecline and predict best clinical benefit earlier than PSAchanges under therapy with abiraterone acetate in bone metastatic castration resistant prostate cancer. BMC Cancer 2016;16:214.

18. Han KS, Hong SJ. Serum alkaline phosphatase differentiates prostate-specific antigen flare from early disease progression after docetaxel chemotherapy in castration-resistant prostate cancer with bone metastasis. J Cancer Res Clin Oncol 2014;140:1769-76.

19. Antonarakis ES, Tagawa ST, Galletti G, et al. Randomized, noncomparative, phase II trial of early switch from docetaxel to cabazitaxel or vice versa, with integrated biomarker analysis, in men with chemotherapy-naive, metastatic, castration-resistant prostate cancer. J Clin
Oncol 2017;35:3181-8.

20. Antonarakis ES, Lu C, Luber B, et al. Clinical significance of androgen receptor splice variant-7 mRNA detection in circulating tumor cells of men with metastatic castrationresistant prostate cancer treated with first- and second-line abiraterone and enzalutamide. J Clin Oncol 2017;35:2149-56.

21. Mori K, Kimura T, Onuma H, et al. Lactate dehydrogenase predicts combined progression-free survival after sequential therapy with abiraterone and enzalutamide for patients with castration-resistant prostate cancer. Prostate 2017;77:1144-50.

22. Armstrong AJ, Saad F, Phung D, et al. Clinical outcomes and survival surrogacy studies of prostate-specific antigen declines following enzalutamide in men with metastatic castration-resistant prostate cancer previously treated with docetaxel. Cancer 2017;123:2303-11.

23. Ueda Y, Matsubara N, Tabata KI, et al. Prostate-specific antigen flare phenomenon induced by abiraterone acetate in chemotherapy-naive patients with metastatic castrationresistant prostate cancer. Clin Genitourin Cancer 2017;15:320-5.

24. Schlack K, Krabbe LM, Fobker M, et al. Early prediction of therapy response to abiraterone acetate using PSA subforms in patients with castration resistant prostate cancer. Int J Mol Sci 2016;17:1520.

25. Burgio SL, Conteduca V, Rudnas B, et al. PSA flare with abiraterone in patients with metastatic castration-resistant prostate cancer. Clin Genitourin Cancer 2015;13:39-43.

26. Angelergues A, Maillet D, Flechon A, et al. Prostatespecific antigen flare induced by cabazitaxel-based chemotherapy in patients with metastatic castrationresistant prostate cancer. Eur J Cancer 2014;50:1602-9.

27. Danila DC, Morris MJ, de Bono JS, et al. Phase II multicenter study of abiraterone acetate plus prednisone therapy in patients with docetaxel-treated castrationresistant prostate cancer. J Clin Oncol 2010;28:1496-501.

28. Danila DC, Heller G, Gignac GA, et al. Circulating tumor cell number and prognosis in progressive castrationresistant prostate cancer. Clin Cancer Res 2007;13:7053-8.

29. Liu R, Cao J, Gao X, et al. Overall survival of cancer patients with serum lactate dehydrogenase greater than 1000 IU/L. Tumour Biol 2016;37:14083-8.

30. Vlachostergios PJ, Oikonomou KG, Gibilaro E, et al. Elevated lactic acid is a negative prognostic factor in metastatic lung cancer. Cancer Biomark 2015;15:725-34.

31. Faloppi L, Bianconi M, Giampieri R, et al. The value of lactate dehydrogenase serum levels as a prognostic and predictive factor for advanced pancreatic cancer patients 
receiving sorafenib. Oncotarget 2015;6:35087-94.

32. Heller G, Fizazi K, McCormack R, et al. The added value of circulating tumor cell enumeration to standard markers in assessing prognosis in a metastatic castrationresistant prostate cancer population. Clin Cancer Res 2017;23:1967-73.

33. Fizazi K, Massard C, Smith M, et al. Bone-related parameters are the main prognostic factors for overall survival in men with bone metastases from castrationresistant prostate cancer. Eur Urol 2015;68:42-50.

34. Fléchon A, Pouessel D, Ferlay C, et al. Phase II study of carboplatin and etoposide in patients with anaplastic progressive metastatic castration-resistant prostate cancer (mCRPC) with or without neuroendocrine differentiation: results of the French Genito-Urinary Tumor Group (GETUG) P01 trial. Ann Oncol 2011;22:2476-81.

35. Nilsson S, Franzen L, Parker C, et al. Bone-targeted radium-223 in symptomatic, hormone-refractory prostate cancer: a randomised, multicentre, placebo-controlled phase II study. Lancet Oncol 2007;8:587-94.

36. Huggins C, Scott WW. Bilateral adrenalectomy in prostatic cancer: clinical features and urinary excretion of 17-ketosteroids and estrogen. Ann Surg 1945;122:1031-41.

37. Scher HI, Morris MJ, Stadler WM, et al. Trial design and objectives for castration-resistant prostate cancer: updated recommendations from the Prostate Cancer Clinical Trials Working Group 3. J Clin Oncol 2016;34:1402-18.

38. Eisenhauer EA, Therasse P, Bogaerts J, et al. New response evaluation criteria in solid tumours: revised RECIST guideline (version 1.1). Eur J Cancer 2009;45:228-47.

39. Roghmann F, Antczak C, McKay RR, et al. The burden of skeletal-related events in patients with prostate cancer and bone metastasis. Urol Oncol 2015;33:17.e9-18.

40. Ryan CJ, Shah S, Efstathiou E, et al. Phase II study of abiraterone acetate in chemotherapy-naive metastatic castration-resistant prostate cancer displaying bone flare discordant with serologic response. Clin Cancer Res 2011;17:4854-61.

41. Johns WD, Garnick MB, Kaplan WD. Leuprolide therapy for prostate cancer. An association with scintigraphic "flare" on bone scan. Clin Nucl Med 1990;15:485-7.

42. Fosså SD, Vaage S, Letocha H, et al. Liposomal doxorubicin (Caelyx) in symptomatic androgenindependent prostate cancer (AIPC)--delayed response and flare phenomenon should be considered. Scand J Urol Nephrol 2002;36:34-9.

43. Scher HI, Halabi S, Tannock I, et al. Design and end points of clinical trials for patients with progressive prostate cancer and castrate levels of testosterone: recommendations of the Prostate Cancer Clinical Trials Working Group. J Clin Oncol 2008;26:1148-59.

44. Scher HI, Warren M, Heller G. The association between measures of progression and survival in castrate-metastatic prostate cancer. Clin Cancer Res 2007;13:1488-92.

45. Rescigno P, Dolling D, Conteduca V, et al. Early posttreatment prostate-specific antigen at 4 weeks and abiraterone and enzalutamide treatment for advanced prostate cancer: an international collaborative analysis. Eur Urol Oncol 2020;3:176-82.

46. Armstrong AJ, Eisenberger MA, Halabi S, et al. Biomarkers in the management and treatment of men with metastatic castration-resistant prostate cancer. Eur Urol 2012;61:549-59.

47. De Laere B, Oeyen S, Van Oyen P, et al. Circulating tumor cells and survival in abiraterone- and enzalutamidetreated patients with castration-resistant prostate cancer. Prostate 2018;78:435-45.

48. Berry WR, Laszlo J, Cox E, et al. Prognostic factors in metastatic and hormonally unresponsive carcinoma of the prostate. Cancer 1979;44:763-75.

49. Scher HI, Jia X, de Bono JS, et al. Circulating tumour cells as prognostic markers in progressive, castration-resistant prostate cancer: a reanalysis of IMMC38 trial data. Lancet Oncol 2009;10:233-9.

50. Terragni R, Morselli-Labate AM, Vignoli M, et al. Is serum total LDH evaluation able to differentiate between alimentary lymphoma and inflammatory bowel disease in a real world clinical setting? PLoS One 2016;11:e0151641.

51. Fitzpatrick JM, Bellmunt J, Fizazi K, et al. Optimal management of metastatic castration-resistant prostate cancer: highlights from a European Expert Consensus Panel. Eur J Cancer 2014;50:1617-27.

52. Halabi S, Armstrong AJ, Sartor O, et al. Prostate-specific antigen changes as surrogate for overall survival in men with metastatic castration-resistant prostate cancer treated with second-line chemotherapy. J Clin Oncol 2013;31:3944-50.

Cite this article as: Schlack K, Krabbe LM, Rahbar K, Isenberg K, Semjonow A, Schrader AJ, Boegemann M. ALP bouncing and LDH normalization in bone metastatic castration-resistant prostate cancer patients under therapy with Enzalutamide: an exploratory analysis. Transl Androl Urol 2021;10(10):3986-3999. doi: 10.21037/tau-20-1117 